\title{
FUNCTIONAL CHANGES IN PERIPHERAL PHAGOCYTES IN RATS WITH LPS- INDUCED PARKINSON'S DISEASE
}

Zhanna OLIYNYK, Educational and Scientific Centre "Institute of Biology \& Medicine", Taras Shevchenko National University of Kyiv, Ukraine, jannaoliynyk@gmail.com (iD https://orcid.org/0000-0003-1614-5856)

Anastasiia MARYNCHENKO, Educational and Scientific Centre "Institute of Biology \& Medicine", Taras Shevchenko National University of Kyiv, Ukraine, whitefox040@gmail.com

(iD https://orcid.org/0000-0002-1956-0177)

Mariya RUDYK, Educational and Scientific Centre "Institute of Biology \& Medicine", Taras Shevchenko National University of Kyiv, Ukraine, rosiente@gmail.com

(iD) https://orcid.org/0000-0003-1252-885X)

Taisa DOVBYNCHUK, Educational and Scientific Centre "Institute of Biology \& Medicine", Taras Shevchenko National University of Kyiv, Ukraine, mtaisa80@gmail.com

(iD) https://orcid.org/0000-0001-7451-6315)

Natalie DZUBENKO, Educational and Scientific Centre "Institute of Biology \& Medicine", Taras Shevchenko National University of Kyiv, Ukraine, $\underline{\mathrm{n} \text { dziubenko@ukr.net }}$

(iD)https://orcid.org0000-0002-2998-4168)

Ganna TOLSTANOVA, Educational and Scientific Centre "Institute of Biology \& Medicine", Taras Shevchenko National University of Kyiv, Ukraine, gtolstanova@gmail.com

(iDhttps://orcid.org/0000-0002-5286-5044)

Received: 26.06.2021, Accepted: 29.12.2021

Research

*Corresponding author Zhanna OLIYNYK

DOI: $10.22531 /$ muglajsci.957174

\begin{abstract}
Sustained neuroinflammation is considered to be a leading contributor to progressive neuron damage of the substantia nigra, leading to the development of Parkinson's disease (PD). Systemic inflammation (SI) correlates with neuroinflammation as PD progresses, and exacerbates neurodegeneration. Phagocytes are key players in both neuroinflammation and SI. SI manifestation in commonly used animal models of PD is an unexplored question. LPS-induced PD is usually used for the study of the inflammation in the PD pathophysiology. The aim of this study was to examine metabolic profile of peripheral phagocytes in rats with LPS-induced PD. LPS-induced PD was accompanied by the neutrophilia, the decrease of circulating lymphocyte proportion, and as a result - by doubling the neutrophil-tolymphocytes ratio. Reactive oxygen species generation was higher in circulating phagocytes from rats with PD (by 1.3 times in neutrophils and by more than 5 times - in monocytes) as compared to control animals. Wherein, phagocytic activity was lower in neutrophils by 1.2 times and in monocytes - by 2.6 times as compared to the control. These data indicate systemic inflammatory process in LPS lesioned rats. Thus, LPS-induced PD reproduces systemic inflammation spread, which is inherent for progressive PD.
\end{abstract}

Keywords: Parkinson's disease, lipopolysaccharide, systemic inflammation, circulating phagocytes, reactive oxygen species, phagocytosis.

Cite

Oliynyk, Z., Marynchenko A., Rudyk M., Dovbynchuk T., Dzubenko N., Tolstanova G., (2021). “Functional Changes in Peripheral Phagocytes in Rats with Lps-Induced Parkinson's Disease", Mugla Journal of Science and Technology, 7(2), 73-78

\section{Introduction}

Parkinson's disease (PD) is a chronic neurodegenerative inflammatory disease. Chronic neuroinflammation is mediated by activated microglia. Another feature of PD which is widely discussed in the literature is systemic inflammation (SI) [1]. According to most sources, PD patients exhibit an elevation of serum proinflammatory 
cytokine (IFN- $\gamma, \mathrm{TNF} \alpha$, IL1 $\beta$, IL17, IL6 etc.) levels irrespective of the presence or absence of comorbidities. Increased levels of some pro-inflammatory cytokines (e.g. IL-1b and IL-6) indicate involvement of peripheral macrophages in SI in PD [2]. In animal models of PD, the depletion of pro-inflammatory peripheral monocyte is associated with disease down-regulation [3]. Hasegawa et al. reported about decreased cytokine release by peripheral immunocytes from PD patients, and about metabolic exhaustion of these cells in persons with advanced disease [4]. Nevertheless, all authors are unanimous that mediators secreted by resident brain phagocytes and phagocytes located at the CNS borders spread to the periphery, and can affect metabolic profile of peripheral cells participating in the development of SI in PD [5]. In such a way, local and systemic inflammatory processes form a vicious cycle (circulus vitiosus) which contributes to perpetuating sustained inflammation in PD [6]. Considering the inflammatory nature of PD and the significance of SI in PD pathophysiology, the investigation of SI in animal models of the disease is crucially important. However, data concerning the manifestation of SI in commonly used animal models of PD are limited and sparse [7]. LPS-induced PD is most faithful animal model for the study of the inflammation in the disease pathophysiology. In these models, LPS induced reactive microgliosis and astrogliosis are accompanied by the release of a plethora of neurotoxic factors which lead to progressive dopaminergic neurodegeneration [8]. The development of reactive microgliosis is registered in different rodent models of PD [9-11]. Previously, we have reported about the association between microglia pro-inflammatory activation and pro-inflammatory metabolic shift of peripheral phagocytes in MPTP- and 6-OHDA-induced PD models $[12,13]$. LPS-induced PD in our experiments was also associated with reactive microgliosis and astrogliosis [14]. This study was aimed to examine metabolic profile of peripheral phagocytes in rats with LPS-induced PD.

\section{Materials and Methods}

\subsection{Experimental Animals}

The size of the papers must be in A4 (210 x $297 \mathrm{~mm})$. The study was performed on male Wistar rats (220-250 g) bred in the vivarium of the Educational and Scientific Centre "Institute of Biology" of Taras Shevchenko National University of Kyiv, Ukraine. The animals were kept in standard conditionswith ad libitumaccess to water and food. Animal protocol was approved by the University Etics Committe according to Animal Welfare Act guidelines. All animal studies were carried out in accordance with the norms established by the Law of Ukraine №3447-IV "On Protection of Animals from Cruelty" as well as in compliance with the standards of the Convention on Bioethics of the Council of Europe's 'Europe Convention for the Protection of Vertebrate Animals' used for experimental and other scientific purposes'(1997), the general ethical principles of animal experiments, approved by the First National Congress on Bioethics Ukraine (September 2001) and other international agreements and national legislation in this field.

Experiment was carried out using three groups of rats ( $n=20$ animals per group). Group 1 -control animals; group 2- sham-operated animals receiving stereotactic $\mathrm{NaCl}$ injection; group 3- LPS-PD animals. LPS-induced hemiparkinsonism was reproduced by stereotactic microinjection of $10 \mu \mathrm{g}$ of LPS (Sigma, USA), dissolved in $2 \mu \mathrm{l}$ of sterile $0.9 \% \mathrm{NaCl}$ ("Infusion", Ukraine) into the substantia nigra ( $\mathrm{AP}=-5.3 ; \mathrm{ML}= \pm 2.0 ; \mathrm{DV}=-7.2)$ [11].

\subsection{Apomorphine Test}

One (I AT) and two (II AT) weeks after the LPS administration pharmacologically induced $(0.5 \mathrm{mg} / \mathrm{kg}$, intraperitoneally, Sigma, USA) rotational movements towards the side contralateral with respect to the toxininjected hemisphere were examined. LPS lesioned animals scoring over $180 \mathrm{rpm}$ were considered as successfully lesioned with $96.6 \%$ dopaminergic neuron (DN) loss. Rats scoring less than $180 \mathrm{rpm}$ were considered as successfully lesioned with $86 \%$ DN loss. Animals without clear contralateral full-body rotation were considered as lesioned with less than 44\% DN loss [15].

\subsection{Immunohistochemistry}

DN degeneration was assessed by immunohistochemical staining with antibodies to tyrosine hydroxylase (TH). Each specimen was accounted semi-quantitatively as to the intensity of immunostaining using a four-point scale, with 0 specifying absence of staining, $1+$ specifying the lowest level of detectable staining and/or nonhomogeneous weak staining, $2+$ specifying moderate homogeneous staining, and $3+$ specifying intense homogeneous staining [16].

\subsection{Hemogram Analysis}

The study of hematological parameters was performed using the analyzer "Particle counter model PCE 210" (ERMA, Japan), adapted for the study of blood cells of rats and mice.

\subsection{Plasma CRP Level Examination}

Plasma was measured for C-reactive protein (CRP) using ELISA Kit (Labcare Diagnostics India Pvt Ltd) according to the manufacturer recommendations.

\subsection{ROS-release Assay}

Phagocyte oxidative metabolism was determined by flow cytometry using 2'7'-dichlorodihydro-fluorescein diacetate (H2DCFDA, Invitrogen) as described previously [17].

\subsection{Phagocytosis Assay}

Phagocytic activity was examined by flow cytometry as described previously [17] FITC-labeled heat-inactivated Staphylococcus aureus Cowan I bacteria (collection of the Department of Microbiology and General Immunology of Taras Shevchenko National University of 
Kyiv) were used as a phagocytosis object. The results were registered as the percentage of cells emitting fluorescence after a defined culture period (phagocytosis percentage, $\mathrm{PhP}$ ) and as phagocytosis index (PhI) that representing the mean fluorescence per one phagocytic cell (ingested bacteria by one cell).

\subsection{Phagocyte Phenotyping}

Phycoerythrin (PE)-labeled anti-CD206, FITC-labelled anti-CD80, and Alexa Fluor-labelled anti-CD86 antibodies (Becton Dickinson, Farmingen, USA) were used for phagocyte phenotyping. Samples were analyzed by FACS Calibur flow cytometer (BD Biosciences, San Jose, CA, USA). The data were analyzed using CELLQuest software (BD; Franklin Lakes, NJ, USA).

\subsection{Statistical Analysis}

All data are expressed as mean \pm SD. For immunological assays, the Student test was used to determine the statistical significance of the difference. A $\mathrm{P}<0.05$ was considered significant. Apomorphine test data analysis was performed using GraphPad Prism5 software (GraphPad Software Inc., San Diego, CA, USA). The onefactor Kraskel-Wallis test and the post-hoc Dunn test were used to determine the reliability of the difference between animal groups. $\mathrm{P}<0.05$ was considered significant.

\section{Results and Discussion}

As mentioned above, LPS-induced PD allows modelling of specific effects of inflammation on DN in clinical course of the disease $[18,19]$. In our experiments, DN loss was evaluated by the results of apomorphine test and by the immunohistochemical staining $\mathrm{TH}$ in the substantia nigra. Apomorphine - a potent dopaminergic D1 and D2 agonist and one of the standard tools in experimental studies of PD in rodents - acts post-synaptically and induce twirl in the opposite contralateral direction (away from the lesioned side) as a result of hyperstimulation of supersensitive DA receptors in the denervated striatum [20]. It's generally accepted that rotation rate more than $5 \mathrm{rpm}$ in apomorphine test in rats means 80\% $\mathrm{TH}$ positive neuronal loss. In animals with neuron loss $\leq 50 \%$, apomorphine-induced rotation is less intensive (0-2 rpm) [21]. In our experiments, rotation rate in LPSlesioned rats was $0.6 \pm 0.4 \mathrm{rpm} 7$ day after and $0.7 \pm 0.6$ rpm 14 days after the disease initiation. It indicates moderate but progressive DN loss in the disease course in animals. Our results agree with the literature data, according to which intracerebral administration of LPS causes moderate progressive decrease in dopamine levels $(<50 \%)$. As a result, apomorphine causes weak contralateral rotational activity (only 0-2 rpm) in lesioned animals [22]. Sensorimotor disregard and anxiety were also observed in LPS lesioned rats by the results of the 'Corridor test' and the 'Open field' test, respectively (data are not presented) [13]. Additionally, DN loss was confirmed by the TH immunostaining (Fig.1).

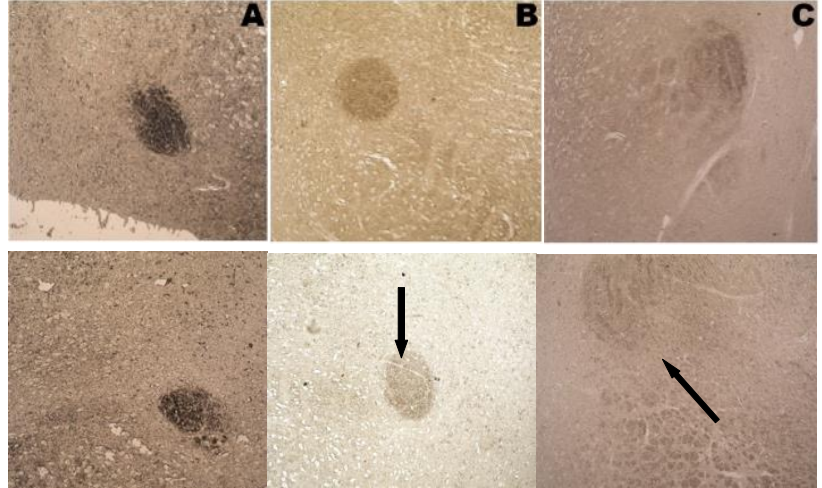

Figure 1.Representative photographs of immunohistological identification of TH-positive neurons in the midbrain of rats with LPS-PD. A - control animal, Bsham-operated animal, C - LPS-lesioned animal. THpositive areas are pointed out with black arrows. $\mathrm{x} 400$.

Semi-quantitative assessment of the results of $\mathrm{TH}$ immunostaining revealed $\sim 65 \%$ reduction of $\mathrm{TH}$ positive neurons in LPS-lesioned animals.

Serum level of CRP is a reliable marker of systemic/peripheral inflammation. Increased serum CRP levels is reported to be inherent and correlate with disease severity in PD subjects [23]. Data concerning CRP level in blood serum of animals with PD are limited. In our experiments, serum level of C-reactive protein in animals with LPS-PD was characterized by significant individual variabilityI. In animals with LPS-PD, a tendency to a slightly higher level of CRP as compared to the control was observed $(189,0+67.0$ vs $138,6+24.4$ $\mu \mathrm{g} / \mathrm{ml})$.

Quantitative and functional indicators of peripheral phagocytes gave a vivid picture of the inflammatory process in the periphery. LPS-PD development was associated with quantitative and metabolic changes of peripheral blood phagocytes. In LPS-PD animals, lymphocyte proportion in peripheral blood was significantly lower than that in control and shamoperated rats at the time of the experiment cessation. Percentage of circulating monocytes didn't differ significantly in animals from control and experimental groups, whereas neutrophils proportion was 1.5 times higher in LPS-PD rats as compared to control animals. (Fig. 2). The development of LPS-PD was associated with a twofold increase in the neutrophil-to-lymphocyte ratio (NLR) as compared to the control, while the monocyteto-lymphocyte ratio (MLR) was only slightly higher than that in control animals (Table 1). Both NLR and MLR are validated markers of the systemic inflammatory process and are widely used to assess and predict the course of many systemic inflammatory processes such as multiple sclerosis, amyotrophic lateral sclerosis, and PD [24]. An increase in NLR and MLR values is associated with an exacerbation of the disease. Fluctuations in NLR correlate to a greater extent with neurological disorders in the case of $\mathrm{PD}$, especially in patients with severe akinetic-rigid syndrome [24]. 
Circulating phagocyte quantitative changes were also accompanied by functional alterations of these cells (Fig.3). Phagocytic activity of circulating neutrophils was decreased as compared to control animals (Fig.3A). Decrease of phagocytic activity is typically inherent for the pro-inflammatory phagocyte metabolic shift, and is associated with the increase of antigen-presenting capacity of these cells [25]. Another important phagocyte metabolic characteristic is the generation of reactive oxygen species, which is involved in the bactericidal and antitumor responses of these cells, and is considered as a marker of M1 metabolic shift [18].

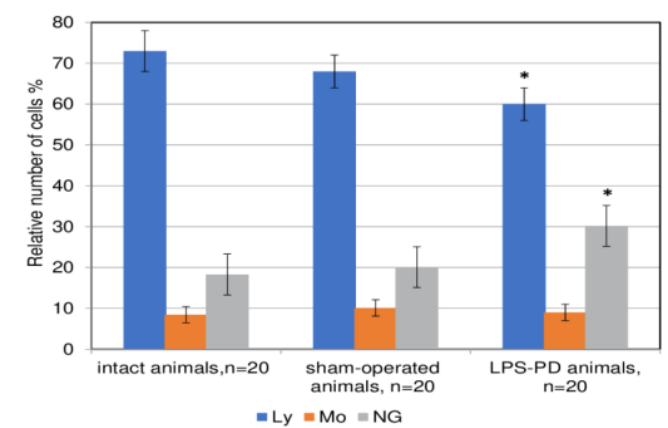

Figure 2. Proportions of lymphocytes, monocytes and neutrophilic granulocytes in rats with LPS-PD. Ly lymphocytes, Mo - monocytes, NG - neutrophilic granulocytes. Note: ${ }^{*}-\mathrm{p} \leq 0.05$, as compared with control animals.

Table 1. Myeloid-to-lymphoid cell ratios in rats with LPSPD

\begin{tabular}{|c|c|}
\hline \multicolumn{2}{|c|}{ neutrophil-to-lymphocyte ratio } \\
\hline control, $\mathrm{n}=20$ & $0.25 \pm 0.04$ \\
\hline $\begin{array}{c}\text { sham-operated, } \\
\mathrm{n}=20\end{array}$ & $0.30 \pm 0.06$ \\
\hline LPS-PD, n=20 & $0.49 \pm 0.04^{*}$ \\
\hline \multicolumn{2}{|c|}{ monocyte-to-lymphocyte ratio } \\
\hline control, n=20 & $0.10 \pm 0,003$ \\
\hline sham-operated, \\
$\mathrm{n}=20$
\end{tabular}

Note: ${ }^{*}$ - $\mathrm{p} \leq 0.05$, as compared with control animals.

Our results revealed significant increase of monocyte ROS generation and moderate increase - in neutrophils from peripheral blood of animals with LPS-PD as compared with control groups (Fig.3B).

We revealed significant increase of monocyte ROS generation and moderate increase - in neutrophils from peripheral blood of animals with LPS-PD as compared with control groups (Fig.3B).
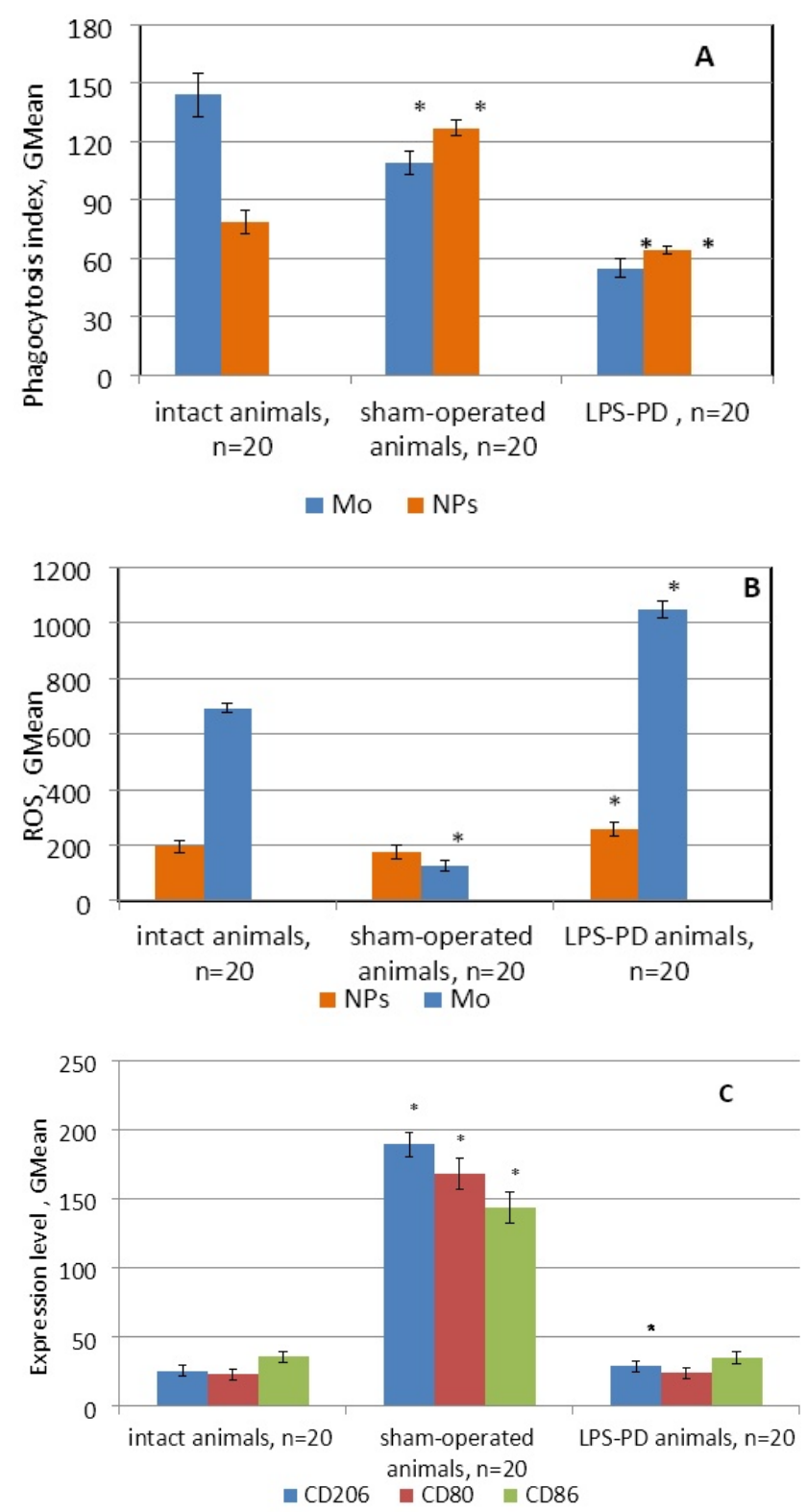

Figure 3. Metabolic and phenotypic caracteristics of circulating monocytes and neutrophils in LPS-PD. Mo monocytes, NPs - neutrophils. A - phagocytic activity, B oxidative metabolism, $\mathrm{C}$ - phenotype. Note: ${ }^{*}-\mathrm{p} \leq 0.05$ as compared with control animals.

The expression level of CD206 and CD80/86 didn't differ significantly in LPS-PD animals from those in control rats (Fig. 3C). Surprisingly, the expression of CD206, as well as the co-stimulatory molecules CD80/86, was significantly up-regulated in sham-operated animals as compared to control rats. Phenotype CD206 ${ }^{\text {high }}$ CD80/86 high is inherent for myeloid suppressor, regulatory cells (Myeloid-Derived Suppressor Cells, MDSC) [26]. It's necessary to note that monocytes from sham-operated animals were also characterized by increased phagocytic activity along with decreased ROS generation indicating antiinflammatory metabolic shift. At the time of the 
experiment, complete wound healing after the surgery was visually observed in sham-operated animals. This allows us to consider anti-inflammatory metabolic and phenotypic profile of circulating phagocytes as a sign of the persistent systemic inflammatory process of a reparative nature in these animals.

\section{Conclusion}

Taken together our data evidence a systemic inflammatory process associated with neutrophilia and pro-inflammatory skew of circulating phagocyte functions in LPS lesioned rats. Therefore, LPS-induced PD model reproduces not only local neuroinflammation, but also systemic inflammation which is inherent for PD.

\section{References}

[1] Ferrari, C.C. and Tarelli, R., "Parkinson'sdisease and systemic inflammation", Parkinsons Dis. 22, 436-813, 2011.

[2] Gundersen, V., "Parkinson's Disease: Can Targeting Inflammation $\mathrm{Be}$ an Effective Neuroprotective Strategy?", Frontiers in neuroscience, 14, 580311, 2021.

[3] Côté, M., Poirier, A., Aubé, B., Jobin, C., Lacroix, S., \&Soulet, D., "Partial depletion of the proinflammatory monocyte population is neuroprotective in the myenteric plexus but not in the basal ganglia in a MPTP mouse model of Parkinson's disease", Brain, behavior, and immunity, 46, 154-167, 2015.

[4] Hasegawa, Y., Inagaki, T., Sawada, M., \& Suzumura, A., "Impaired cytokine production by peripheral blood mononuclear cells and monocytes/macrophages in Parkinson's disease", Acta neurologica Scandinavica, 101(3), 159-164, 2000.

[5] Ivan, D. C., Walthert, S., Berve, K., Steudler, J., \& Locatelli, G., "Dwellers and Trespassers: Mononuclear Phagocytes at the Borders of the Central Nervous System", Frontiers in immunology, 11, 609921, 2021.

[6] Ortiz, G. G., González-Usigli, H., Pacheco-Moisés, F. P., Mireles-Ramírez, M. A., Sánchez-López, A. L., TorresSánchez, E. D., ... González, V. S., "Physiology and pathology of neuroimmunology: Role of inflammation in parkinson's disease", Physiology and Pathology of Immunology. IntechOpen, 2017.

[7] Gasparotto, J., Ribeiro, C. T., Bortolin, R. C., Somensi, N., Rabelo, T. K., Kunzler, A., Souza, N. C., Pasquali, M., Moreira, J., \&Gelain, D. P., "Targeted inhibition of RAGE in substantia nigra of rats blocks 6-OHDAinduced dopaminergic denervation", Scientific reports, 7(1), 8795, 2017.

[8] Liu, M., \& Bing, G., "Lipopolysaccharide animal models for Parkinson's disease", Parkinson's disease, 327089, 2011.

[9] Yıldızhan, K., Nazıroğlu, M. “Glutathione Depletion and Parkinsonian Neurotoxin MPP+-Induced TRPM2 Channel Activation Play Central Roles in Oxidative
Cytotoxicity and Inflammation in Microglia", Molecular neurobiology, 57(8), 3508-3525, 2020.

[10] Fricke, I.B., Viel, T., Worlitzer, M.M., Collmann, F.M., Vrachimis, A., Faust, A., Wachsmuth, L., Fabe, C., Dollé, F., Kuhlmann, M.T., Schäfers, K., Hermann, S., Schwamborn, J.C., Jacobs, A.H. "6-hydroxydopamineinduced Parkinson's disease-like degeneration generates acute microgliosis and astrogliosis in the nigrostriatal system but no bioluminescence imaging-detectable alteration in adult neurogenesis", The European journal of neuroscience, 43(10),1352-1365, 2016.

[11] Hoban, D. B., Connaughton, E., Connaughton, C., Hogan, G., Thornton, C., Mulcahy, P., Moloney, T. C., \& Dowd, E., "Further characterisation of the LPS model of Parkinson's disease: a comparison of intra-nigral and intra-striatal lipopolysaccharide administration on motor function, microgliosis and nigrostriatal neurodegeneration in the rat", Brain, behavior, and immunity, 27(1), 91-100, 2013.

[12] Rudyk, M., Hurmach, I., Svyatetska, V., Prysyazhnyuk, O., Dovbynchuk, T., Skachkova, O., TolstanovaG., Skivka, L., "Peripheral phagocyte characteristics as markers of systemic inflammation in rats with different stages of Parkinson's disease", EUROPEAN JOURNAL OF CLINICAL INVESTIGATION (Vol. 48, pp. 73-73). 111 RIVER ST, HOBOKEN 07030-5774, NJ USA: WILEY 89, 2018.

[13] Rudyk, M. P., Opeida, I. V., Svyatetska, V. M., Prysiazhniuk, A. I., Dovbynchuk, T. V., Khranovska, N. M., Tolstanova G.M., Skivka, L. M., "Microglia and circulating phagocyte metabolic profile in rats with MPTP-induced Parkinson's disease and concomitant ulcerative colitis", EUROPEAN JOURNAL OF CLINICAL INVESTIGATION (Vol. 47, pp. 151-152). 111 RIVER ST, HOBOKEN 07030-5774, NJ USA: WILEY, 2017.

[14] Oliynyk, Z., Senchylo, N., Dovbynchuk, T., Stepanenko, S., \& Guzik, M., "Reactive astrogliosis in rats with LPS-induced Parkinson's disease", Bulletin of Taras Shevchenko National University of KyivBiology, 80(1), 19-25, 2020.

[15] Talanov, S.A., Oleshko, N.N., Tkachenko, M.N., Sagach, V.F., "Pharmacoprotective influences on different links of the mechanism underlying 6hydroxydopamine-induced degeneration of nigrostriatal dopaminergic neurons", Neurophysiology 38 (2), 128-133, 2006.

[16] Walsh, S., Finn, D. P., \& Dowd, E., "Time-course of nigrostriatal neurodegeneration and neuroinflammation in the 6-hydroxydopamineinduced axonal and terminal lesion models of Parkinson's disease in the rat", Neuroscience, 175, 251-261, 2011.

[17] Rudyk, M., Pozur, V.V., Voieikova, D., Hurmach, Y., Khranovska, N., Skachkova, O., Svyatetska, V., Fedorchuk, O.G., Skivka, L., Berehova, T.V., \&Ostapchenko, L., "Sex-based differences in phagocyte metabolic profile in rats with 
monosodium glutamate-induced obesity", Scientific Reports, 8, 2018.

[18] Deng, I., Corrigan, F., Zhai, G., Zhou, X., \&Bobrovskaya, L., "Lipopolysaccharide animal models of Parkinson's disease: Recent progress and relevance to clinical disease", Brain, Behavior, \& Immunity - Health, Volume 4, 2020.

[19] Björklund, A., \& Dunnett, S. B, "The Amphetamine Induced Rotation Test: A Re-Assessment of Its Use as a Tool to Monitor Motor Impairment and Functional Recovery in Rodent Models of Parkinson's Disease", Journal of Parkinson's disease, 9(1), 17-29, 2019.

[20] Iancu, R., Mohapel, P., Brundin, P., Paul, G. "Behavioral characterization of a unilateral 6-OHDAlesion model of Parkinson's disease in mice", Behavioural brain research, 162(1), 1-10, 2005.

[21] Miyanishi, K., Choudhury, M. E., Watanabe, M., Kubo, M., Nomoto, M., Yano, H., \& Tanaka, J., "Behavioral tests predicting striatal dopamine level in a rat hemi-Parkinson's disease model.", Neurochemistry international, 122, 38-46, 2019.

[22] Eidson, L. N., Kannarkat, G. T., Barnum, C. J., Chang, J., Chung, J., Caspell-Garcia, C., Taylor, P., Mollenhauer, B., Schlossmacher, M. G., Ereshefsky, L., Yen, M., Kopil, C., Frasier, M., Marek, K., Hertzberg, V.
S., \& Tansey, M. G., "Candidate inflammatory biomarkers display unique relationships with alphasynuclein and correlate with measures of disease severity in subjects with Parkinson's disease", Journal of neuroinflammation, 14(1), 164, 2017.

[23] Choi, S. J., Hong, Y. H., Kim, S. M., Shin, J. Y., Suh, Y. J., \& Sung, J. J., "High neutrophil-to-lymphocyte ratio predicts short survival duration in amyotrophic lateral sclerosis", Scientific reports, 10(1), 428, 2020.

[24] Umehara, T., Oka, H., Nakahara, A., Matsuno, H., \& Murakami, H., "Differential leukocyte count is associated with clinical phenotype in Parkinson's disease", Journal of the neurological sciences, 409, 116638, 2020.

[25] Schulz, D., Severin, Y., Zanotelli, V., \& Bodenmiller, B., "In-Depth Characterization of Monocyte-Derived Macrophages using a Mass Cytometry-Based Phagocytosis Assay", Scientific reports, 9(1), 1925, 2019.

[26] Veglia, F., Perego, M., \& Gabrilovich, D., "Myeloidderived suppressor cells coming of age", Nature immunology, 19(2), 108-119, 2018. 